\section{La Révolution française}

Cahiers de l'Institut d'histoire de la Révolution française

\section{$6 \mid 2014$}

La Révolution ou l'invention de la femme et de l'homme nouveaux

\title{
Gilles Bertrand, Pierre Serna (dir.), La République en
}

voyage, 1770-1830

Rennes, Presses Universitaires de Rennes, 2013, 442 pages

\section{Dominique Waquet}

\section{OpenEdition \\ Journals}

Édition électronique

URL : http://journals.openedition.org/lrf/1146

DOI : $10.4000 /$ Irf. 1146

ISSN : $2105-2557$

Éditeur

IHMC - Institut d'histoire moderne et contemporaine (UMR 8066)

Référence électronique

Dominique Waquet, « Gilles Bertrand, Pierre Serna (dir.), La République en voyage, 1770-1830 », La

Révolution française [En ligne], 6 | 2014, mis en ligne le 28 septembre 2014, consulté le 22 septembre 2020. URL : http://journals.openedition.org//rf/1146 ; DOI : https://doi.org/10.4000/Irf.1146

Ce document a été généré automatiquement le 22 septembre 2020.

(c) La Révolution française 


\section{Gilles Bertrand, Pierre Serna (dir.), La République en voyage, 1770-1830}

Rennes, Presses Universitaires de Rennes, 2013, 442 pages

Dominique Waquet

\section{RÉFÉRENCE}

Gilles Bertrand, Pierre Serna (dir.), La République en voyage, 1770-1830, Rennes, Presses Universitaires de Rennes, 2013, 442 pages

1 La République en voyage. Provocation ou antiphrase ? Dans nos souvenirs, la République, bien que toujours triomphante, ne "voyageait " pas réellement. Dessinée ${ }^{1}$, gravée ${ }^{2}$ ou statufiée ${ }^{3}$, elle restait toujours hiératique sur son char, immobile. Cependant, une fois sa légitimité définitivement assurée, reconnaissons que sur ce véhicule devenu animé, elle a souhaité s'implanter en région, rallier Bordeaux, triompher encore sur la place des Quinconces et se poser pour la postérité dans la Fontaine des Girondins ${ }^{4}$.

2 Mais ôtons à La République son voile métonymique. L'ouvrage nous donne alors à voir des hommes et de femmes aux positions républicaines, affirmées ou en devenir, des sujets de divers royaumes, mais qui auraient bien mérité d'être républicains, et tous et toutes en déplacement ou en itinérance plus ou moins au nom de la République. Nous y découvrons aussi d'autres hommes et d'autres femmes qui, sans être républicains, ni de cœur ni d'engagement, ont été poussés à l'errance, souvent par une idéologie républicaine exacerbée, sinon dévoyée.

3 Car convoquer le voyage dans le titre de l'ouvrage, c'est annoncer que tous ses héros sont décrits en mouvement: mission officielle, déplacement privé, pérégrination, périple, marche, apodémie, errance, fuite. Voyage a été pris, ici, dans un sens extensif voire allégorique puisque même la lecture, la traduction sont vues comme des voyages. Cet imposant recueil serait-il donc un long journal de bord, le récit d'épopées politiques ou même l'itinéraire allégorique du Paris des Lumières vers ... la Cité Idéale ${ }^{5}$ ? 
Concrètement, La République en voyage est la somme des communications du colloque tenu sur ce thème à Vizille en septembre 2010. Mais les deux co-directeurs du colloque et de l'ouvrage « n'ont pas souhaité reprendre l'ordre des communications données lors du colloque ${ }^{6}$. " Confrontés à une matière riche et diversifiée, ils ont voulu la rebrasser et la discipliner " selon un cadre chronologique précis ${ }^{7}$ » et l'ont finalement organisée suivant une taxinomie hautement élaborée et finement libellée ${ }^{8}$. Ainsi, encadrées d'une introduction, d'une préface, d'une conclusion et d'une postface, les 22 contributions de 23 auteurs, assistés de deux traducteurs, ont donc finalement été ventilées en 10 chapitres regroupés eux-mêmes en « cinq temps, de respiration et d'intensité variable ${ }^{9}$ $\gg$ :

1. La mise en place d'un voyage républicain

2. Révolution, conflits et voyages mouvementés

3. La République traduite, la république en transfert

4. Le voyage, fabrique des savoirs républicains

5. Au bout du voyage, héritage d'une génération

5 A la fin de l'ouvrage le lecteur trouvera l'habituelle présentation des auteurs et l'index des noms propres, opportunément catégorisé. Il encourt cependant la légère frustration de ne pas trouver, dans ce recueil consacré aux voyages, un index des noms de lieux.

6 La problématique de La République en voyage, par un renversement conceptuel et stylistique à visée opératoire, se transforme, dès l'introduction de l'ouvrage, en problématique du « voyage républicain ». Et même ainsi, comme G. Bertrand le souligne d'emblée, "la question de la définition d'un voyage républicain est [...] complexe ${ }^{10}$.» Sans aucun doute. Mais après avoir lu l'ensemble des communications de ce recueil, il n'est pas illégitime de se demander s'il était vraiment utile de chercher à qualifier un voyage de "républicain» au motif qu'il se déroule à une époque de l'Histoire où s'instaure un système politique déclaré comme "républicain », tant en France que dans quelques états européens et aux Etats-Unis d'Amérique. De même, on peut se demander s'il fallait s'interroger sur la nature "républicaine » des sources littéraires exploitées par les contributeurs du colloque. En effet, « pas plus qu'il n'existe un genre stabilisé de récit du voyage, il n'existe un type d'écriture propre à l'expérience républicaine du voyage $^{11}$.» Enfin, est-il nécessaire de quitter son fauteuil pour voyager? Non, dit Kant, cité par Daniel Roche dans ses incontournables et, semblerait-il déjà oubliées, Circulations ${ }^{12}$. Oui, dit Rousseau, voyageur lui-même, pour parfaire l'éducation de son Émile ${ }^{13}$.

7 Plus rousseauiste que kantien, mais pour cette fois seulement, je vais donc tenter de dire d'abord qui sont ces voyageurs qui osent ou doivent se bouger en ces temps républicains et pourquoi ils voyagent. Les résultats, les facteurs principaux de réussite ou d'échec de leurs déplacements ressortiront ensuite naturellement des travaux des contributeurs au colloque. Et je n'oublierai évidemment pas les contributions et apports de celles et ceux qui ont travaillé sur les traductions et autres productions écrites, sans voyage sous-jacent. 


\section{Qui voyage en ces temps républicains ? Dans ce panel, principalement des officiels sans mission officielle}

Les voyageurs dont les pérégrinations sont rapportées dans l'ouvrage ne sont et ne peuvent être représentatifs - au sens statistique du terme - de tous ceux et celles qui ont voyagé durant cette période, ni même de tous ceux et celles qui, ayant voyagé, ont laissé une trace écrite de leurs déplacements ${ }^{14}$. Malgré cela, ceux et celles que j'ai envie de nommer héros et héroïnes du colloque de Vizille sont suffisamment différents les un(e)s des autres pour autoriser un embryon de typologie, même s'ils sont, évidemment, tous lettrés, cultivés et en plus curieux, par goût ou par nécessité.

Cette typologie des héros voyageurs va permettre de voir si, selon leur catégorie, ils ont des expériences de voyages différentes ou similaires, s'ils diffèrent ou se retrouvent dans la vision qu'ils, elles, portent sur ces voyages, dans la façon de les raconter, dans leur engagement politique ou idéologique, ...

\section{a) Le statut socio-professionnel}

Les premières différences pourraient provenir de la position socio-professionnelle des héros. Ces voyageurs du temps de la République, rassemblés dans le panel du colloque, sont des personnages « officiels » ou des « personnes privées » (Tableau 1).

1) Parmi les "officiels", élus, fonctionnaires, militaires, titulaires d'offices, mais aucune femme bien sûr, voici :

- les français Barère (présenté dans l'article de M. Bouyssy), Bourgoing (J.-R. Aymes), Bourienne (D. Tinková), Brissot (A. Jourdan), Gerlain (F. Régent), des Girondins proscrits dont Barbaroux, Brissot, Buzot, Cussy, Guadet, Louvet, Pétion et une dizaine d'autres (G. Mazeau), Joubert (D. Tinková), Lafayette (E. Liris), le duc de la Force (D. Tinková), Malesherbes (A. Jourdan), Roland (A. Jourdan), A. Thouin (P.-Y. Lacour et M. Van StrienChardonneau).

- les étrangers Clavière (Suisse, R. Markovits), Foscolo et ses émules (Italiens, présentés par 0. Müller), J. Harris, Lord Malmesbury (Anglais, S. Kleinman), d'Ivernois (Suisse, R. Markovits), T.W. Tone (Irlandais, S. Kleinman), Oraczewski (Polonais, A. Janiszewska), Paulus (Hollandais, A Baggerman, R. Dekker), G. Pepe (Italien, A.-M. Rao), van Eck (Hollandais, A Baggerman, R. Dekker).

2) Les « personnes privées » sont:

- des hommes, Laugier (A. Jourdan), Swinburne (Anglais, S. Kleinman),

- des femmes, «A Lady» (Anglaise, S. Kleinman), Mary Pinckney (Américaine, S. Kleinman), de nombreuses religieuses, entre autres les sœurs Ave, Barat, Gauchat, Kervingant, Miel (N. Pellegrin), Helen Williams (Anglaise, B. Gainot).

Tableau 1

\begin{tabular}{|l|l|l|l|l|}
\hline Panel des voyageurs sujets du colloque & « Officiels » & \multicolumn{2}{|l|}{ « Personnes privées » } & TOTAL \\
\hline & Hommes & Hommes & Femmes & \\
\hline Français & 31 & 1 & 7 & $\mathbf{3 9}$ \\
\hline
\end{tabular}




\begin{tabular}{|l|l|l|l|l|}
\hline Etrangers & 13 & 1 & 3 & 17 \\
\hline TOTAL & 44 & 2 & 10 & 56 \\
\hline
\end{tabular}

N.B. Ce dénombrement des voyageurs concerne les principaux personnages cités dans les articles. II n'a pas de prétention à l'exhaustivité et donne un ordre de grandeur.

11 La segmentation de la soixantaine de héros du panel selon leur statut socioprofessionnel montre que les traces de voyages historiographiquement exploitables, et exploitées dans ce colloque, sont essentiellement laissées par des hommes investis de fonctions officielles. Et peu importe ici qu'ils soient français ou étrangers. Les hommes non investis d'un statut officiel n'ont peut-être pas suffisamment écrit sur leurs voyages pour avoir une plus grande place dans un ouvrage comme celui-ci, ou alors auraient-ils moins voyagé ? Les femmes, plus prolixes et fines observatrices, avaient manifestement beaucoup à dire sur leurs déplacements internationaux.

La lecture attentive des contributions de l'ouvrage ne donne pas à penser que le statut socio-professionnel ait eu une influence sur les supports narratifs des héros ni sur la richesse et la diversité des situations et expériences rapportées.

\section{b) En mission ou « autonomes » (Tableau 2)}

Parmi ces héros, quelques-uns voyagent sur ordre de mission. On trouve dans le panel, comme voyageurs-écrivains en mission :

- des diplomates : Bourgoing, le Français, en Espagne, et à Paris J. Harris, Lord Malmesbury, l'Anglais ainsi que Oraczewski, le Polonais ;

- des négociateurs: Paulus et Van Eck, les patriotes hollandais à Paris, ainsi que Tone, l'Irlandais ;

- des militaires : Pepe, l'Italien en France et en Espagne ;

- des envoyés spéciaux : Thouin, le botaniste français, aux Provinces-Unies et en Italie.

J'aurais presque eu envie de leur adjoindre l'épistolière anonyme "A Lady ", en mission par alliance, car vraisemblablement épouse de l'aide de camp du diplomate anglais J. Harris.

Et puis, il y a les autres, que je regroupe, pour les besoins de la segmentation, dans une catégorie un peu fourre-tout de voyageurs « autonomes ». Cette dernière dénomination n'implique pas nécessairement qu'ils voyagent de leur plein gré, ou par curiosité intellectuelle et encore moins pour humer l'air du temps républicain.

Tableau 2

\begin{tabular}{|l|l|l|l|}
\hline Panel des voyageurs sujets du colloque & « en mission » & « autonomes » & TOTAL \\
\hline « Officiels» & 8 & 36 & $\mathbf{4 4}$ \\
\hline «Personnes privées » & 0 & 12 & $\mathbf{1 2}$ \\
\hline TOTAL & $\mathbf{8}$ & $\mathbf{4 8}$ & $\mathbf{5 6}$ \\
\hline
\end{tabular}


Si tous les voyageurs en mission sont des officiels, tous les officiels du panel ne voyagent pas «en mission", et de très loin. Ils se retrouvent alors, nettement plus nombreux, dans le groupe des voyageurs « autonomes ». Dans tous les cas, les écrits des officiels en mission ne diffèrent guère dans leur support, leur agencement et leurs contenus de ceux des voyageurs qui ont décidé seul(e)s de se lancer dans un déplacement dont les contributeurs de l'ouvrage nous relatent les péripéties et observations. Il va falloir chercher si les mobiles du voyage et ses résultats discriminent mieux les héros du colloque.

\section{Voyages réussis et échecs, en et hors de France}

S'il parait assez vain de chercher à discriminer finement les voyages selon des causes et des objectifs qualifiés de "républicains", il ne fait pas de doute que le contexte de l'émergence d'une nouvelle organisation de la société sous la forme d'une République a suscité bon nombre des voyages rapportés dans l'ouvrage, mais pas tous.

Les voyages qui nous sont rapportés dans l'ouvrage ont été entrepris pour des raisons variées, par exemple une mission officielle comme cela vient d'être dit, mais ce n'est que la minorité des causes de voyage. On trouve, dans ce recueil, des missions officieuses, des missions auto-attribuées, des évasions, des mutineries, des fuites volontaires et des fuites provoquées, ainsi que du tourisme, des parenthèses culturelles et des visites à de vieux amis. Même si ces derniers épisodes sont, dans cet ouvrage, annexes à l'objectif principal du voyage, ils n'en sont pas moins bien réels et rapportés par les contributeurs.

\section{a) Tous ces voyages sont-ils des réussites ? Pas vraiment !}

19 Il est tentant de chercher à quantifier le taux de réussite des voyages en partant de leur objectif et en regardant si l'objectif a été atteint. Pour ce faire, il faut simplifier les données. Les contributeurs ont souvent, dans leurs articles respectifs, présenté plusieurs voyageurs ayant le même objectif ou pris dans la même situation. Pour avoir une idée d'ensemble Il semble donc justifié de mener la quantification au niveau de la contribution et pas à celui du voyageur. Supposons qu'à chaque contribution de l'ouvrage, hormis les quatre dans lesquelles on ne voyage pas, corresponde un seul objectif pour le voyage relaté. Soit explicitée, soit suggérée, la bonne ou la mauvaise réalisation de l'objectif apparaît dans la contribution.

La réussite de la mission diplomatique de Bourgoing, par exemple, est évidente ${ }^{15}$. Envoyé officiellement en poste à Madrid en 1777, il se donne, en plus, à lui-même la mission de visiter le pays pour mieux le comprendre, tant ce qu'il en a lu lui semble contradictoire. Il veut se faire son opinion. Il la forge et il l'écrit. A l'opposé, s'avère non moins patent, l'échec de la tentative d'implantation dans l'empire autrichien de plusieurs émigrés français, représentatifs de la « monarchie en fuite ${ }^{16}$.»

21 En classant les résultats des voyages, évalués sous ma seule responsabilité, comparés aux objectifs, d'une part sur un continuum succès-échec et d'autre part selon les territoires où se déroule principalement le récit, je constate que les histoires choisies par les contributeurs ne sont pas toutes des success stories. 
Je n'ai pu retenir que 17 articles de l'ouvrage sur 22 (Tableau 3). Quatre articles en effet ne parlent pas de voyages réalisés et le cinquième ne met en avant ni héros voyageurs (à part Louis XIV et Napoléon, hors concours), ni objectifs dans les déplacements qui y sont cependant évoqués ${ }^{17}$. Pour passer de 17 articles à 23 résultats dans le tableau cidessous, 6 articles ont été comptés deux fois pour signaler les différents résultats d'un même voyage selon ses étapes. Dans ce tableau, faute de place, les noms des auteurs des articles représentent voyages et voyageurs.

Tableau 3

\begin{tabular}{|c|c|c|c|c|c|}
\hline $\begin{array}{l}\text { Résultats } \\
\text { des voyages } \\
\text { par article }\end{array}$ & Succès & Succès mitigé & Échec relatif & Échec & TOTAL \\
\hline $\begin{array}{l}\text { Voyages en } \\
\text { France }\end{array}$ & Janizeswka, Gainot, & Pellegrin, Rao & $\begin{array}{l}\text { Baggerman- } \\
\text { Decker, } \\
\text { Régent, Liris }\end{array}$ & $\begin{array}{l}\text { Mazeau } \\
\text { Kleinman, } \\
\text { Bouyssy }\end{array}$ & 10 \\
\hline $\begin{array}{l}\text { Voyages ou } \\
\text { étapes hors } \\
\text { de France }\end{array}$ & $\begin{array}{l}\text { Jourdan, Aymes, } \\
\text { Gainot, Lacour, Van } \\
\text { Strien-Chardonneau, } \\
\text { Liris }\end{array}$ & $\begin{array}{l}\text { Pellegrin, } \\
\text { Régent }\end{array}$ & Bouyssy & $\begin{array}{l}\text { Markovits, } \\
\text { Tinkova, } \\
\text { Müller }\end{array}$ & 13 \\
\hline TOTAL & 8 & 5 & 4 & 6 & 23 \\
\hline
\end{tabular}

N.B. Les noms soulignés concernent les auteurs cités deux fois, matérialisant ainsi les résultats différents et propres aux étapes principales des voyages de leurs héros/héroïnes en France et hors de France.

Dernière approche du taux de réussite du voyage tel que j'essaye d'en rendre compte en fonction de son objectif (Tableau 4). Considérons qu'à chaque thème du ou des voyages rapportés par chaque article soit associé principalement l'un des objectifs suivants : observer, fuir, agir, négocier. La répartition des objectifs par résultat s'établit ainsi :

Tableau 4

\begin{tabular}{|l|l|l|l|l|l|}
\hline Résultats des voyages par article & Succès & Succès mitigé & Échec relatif & Échec & TOTAL \\
\hline Observer & 4 & & & & 4 \\
\hline Fuir & 1 & 3 & 3 & 4 & 11 \\
\hline Agir & 3 & 2 & & 1 & 6 \\
\hline Négocier & & & 1 & 1 & 2 \\
\hline TOTAL & 8 & 5 & 4 & 6 & 23 \\
\hline
\end{tabular}

Il sera difficile d'affirmer que le succès est dans la fuite! Une des clés du succès d'un voyage réside en effet dans la qualité du réseau alerté et sollicité avant et pendant le 
voyage. Et quand on fuit, surtout en période de révolutions et d'agitations militaires, il est bien difficile de mobiliser les bons réseaux, quand encore ils existent là où l'on va!

\section{b) Les réseaux " sociaux », facteur nécessaire de réussite du voyage entre 1770 et 1830}

Il est banal, sinon trivial - comme disent les mathématiciens - de constater que les élites se connaissent, se fréquentent et s'entraident. C'est vrai des people d'aujourd'hui. C'était évidemment vrai et vérifiable à l'époque de cette étude. Qui veut voyager avec profit doit disposer de bonnes têtes de réseaux. Elles prépareront les relais, ouvriront les portes et d'abord les leurs. C'est bien le drame des proscrits, comme cela nous est opportunément rappelé par exemple par G. Mazeau pour les Girondins ${ }^{18}$ et par N. Pellegrin pour les religieuses ${ }^{19}$, que de ne pas pouvoir compter sur des réseaux solides. Soit les réseaux n'existent pas, soit ils existent mais craignent les accusations de complicité, les délations et les condamnations qui s'en suivent.

Il n'est pas possible ici, faute de temps et de place, de tracer la cartographie des réseaux auxquels appartiennent les héros du recueil. Toutefois les données existent. En attendant de disposer d'un Facebook ${ }^{\mathrm{m}}$ 1790-1800 reconstitué, je livre un double indicateur de popularité des voyageurs, cités dans le recueil ${ }^{20}$ (Tableau 4), excluant volontairement les abondantes citations des célébrités d'influence et d'action comme Montesquieu, Napoléon, Rousseau ou Voltaire, ici non voyageurs.

Tableau 5

\begin{tabular}{|l|l|l|}
\hline $\begin{array}{l}\text { Personnage voyageur cité dans } \\
\text { l'ouvrage }\end{array}$ & $\begin{array}{l}\text { Nombre de pages où est } \\
\text { cité }\end{array}$ & $\begin{array}{l}\text { Nombre d'articles où est } \\
\text { cité }\end{array}$ \\
\hline J.-P. Brissot & 26 & 6 \\
\hline G. de Lafayette & 23 & 6 \\
\hline T. W. Tone & 20 & 4 \\
\hline E. Clavière & 17 & 5 \\
\hline A. Thouin* & 14 & 4 \\
\hline T. Paine & 12 & 5 \\
\hline J. Pétion & 11 & 3 \\
\hline J.-B. Louvet & 10 & 3 \\
\hline J.-M. Roland & 7 & 2 \\
\hline
\end{tabular}

* Un biais structurel amplifie le score de Thouin puisque deux articles consécutifs lui sont consacrés.

Curieusement, car les contributeurs du colloque ne se sont sans doute pas donné le mot pour évoquer massivement l'inspirateur de la Gironde, Brissot, super-héros de cet ouvrage, arrive en tête du palmarès des citations pour voyages "républicains". 
Lorsque qu'il prend son premier départ pour l'étranger, vers la Suisse en 1782, il n'a que 28 ans mais est déjà connu, et dispose d'un réseau de relations incroyablement étendu et fourni ${ }^{21}$. Dans son réseau, on trouve assez tôt ceux qui vont l'accompagner dans son aventure politique et dans son odyssée humaine. Ils sont présents en force dans les voyageurs évoqués à Vizille. Cette présence de Brissot et celle de plusieurs de ses amis serait-elle alors une valeur ajoutée collatérale du colloque permettant de revisiter son influence ${ }^{22}$ ?

Toutefois, il n'a pas suffit ni à Brissot pour sauver sa tête, ni à Lafayette pour vivre tranquille en France ${ }^{23}$, de compter, ici et ailleurs, d'innombrables «amis » de tous horizons, de se voir considérés en France, en Europe et aux Etats-Unis comme des références, des "têtes de réseaux ». Le réseau est une condition nécessaire de la réussite sociale et de celle des déplacements spatio-temporels. Il n'en est pas une condition suffisante.

\section{Les idées voyagent-elles par les traductions?}

Quatre contributions regroupées dans la troisième partie de l'ouvrage sous la bannière "République traduite, république en transfert » ne traitent pas de voyages en tant que déplacements physiques d'êtres humains, mais de "voyages" correspondants à des transformations/adaptations linguistiques d'idées et de concepts. Si j'ai bien compris, c'est l'acte de traduire qui serait en soi un "voyage». À moins que ce ne soit le déplacement de l'objet support de la traduction, livre, lettre, déclaration, code, ... depuis le traducteur vers le lecteur de la traduction, où qu'il se trouve?

Ce n'est pas le lieu ici de débattre de l'éventuelle nature politique de l'acte de traduction ${ }^{24}$, et je ne suis pas qualifié pour cela ${ }^{25}$. Mais si l'on considère que le voyage est vision de l'autre, de son cadre de vie et de pensée ainsi que de l'autre dans ${ }^{26}$ son cadre de vie, l'imprégnation culturelle de la différence et l'enrichissement qui en découle ne peuvent venir que du transport, pas d'une image.

Au-delà de l'interrogation suscitée par le positionnement de cette greffe éditoriale, il est cependant assuré que ces quatre contributions sont riches, passionnantes et fort instructives.

Ainsi, la façon dont Madison, futur président des Etats-Unis, réfléchit dès 1784 à une meilleure organisation de la jeune République est à cet égard exemplaire ${ }^{27}$. Il ne voyage pas physiquement, ce qui ne lui aurait pas été d'une grande utilité pour connaître et comprendre Platon ou Montesquieu. Il se documente et lit. On voit bien ici comment la qualité de la réflexion sur l'organisation politique de la société vient de la profondeur et de l'étendue des études livresques, des discussions et des échanges épistolaires avec ses pairs. Etudier et voyager sont des concepts différents, même s'ils sont habilement et souvent réunis, aujourd'hui par exemple, sous le vocable englobant, séduisant bien que souvent trompeur, de " voyages d'étude ».

Donc Madison étudie dans le silence de son salon de Montpelier (Comté d'Orange, Virginie). Il veut recomposer la Constitution de la confédération américaine pour calmer les tiraillements, le mot est faible, entre le "pouvoir " fédéral et les états confédérés, frétillants dans leur autonomie fraîchement arrachée à l'Angleterre. Il lit, puisque l'action nait de la réflexion. Il propose. De ses études naîtront des principes. Des principes mis en œuvre sur le sol américain naîtront une constitution et des 
pratiques politiques. Ce sont ces pratiques que viendront observer les voyageurs, héros des autres chapitres du recueil, dont Brissot en 1788. Et c'est bien lui, et d'autres voyageurs, comme Lafayette, qui ramèneront vers la France principes et pratiques constitutionnelles américaines.

Quelques mots, enfin, sur une proposition du conventionnel Portiez, député de l'Oise. Sa présentation, qui aurait mieux trouvé sa place dans la quatrième partie de l'ouvrage, nous ramène bien au voyage, cette fois comme complément à l'éducation des jeunes gens de la République ${ }^{28}$. Face aux projets ultra-simplificateurs des réformes de l'enseignement, dont la suppression des collèges, Portiez en juillet 1794 remet au goût du jour républicain l'ars apodemia, le Grand Tour, dont l'utilité n'était pas et n'est plus à démontrer ${ }^{29}$. Puisque l'Etat doit tout organiser autant que ce soit utile, à l'éducation, bien entendu, et à l'amélioration de la conscience républicaine de ceux qui auront été sélectionnés pour visiter la France et quelques pays alentour. Le contributeur insiste dans sa conclusion sur cette " collectivisation d'une pratique jusqu'ici essentiellement individuelle, [dans laquelle] réside l'intérêt du plan de Portiez ${ }^{30}$. » Faut-il se réjouir ou regretter que cette collectivisation n'ait pu voir le jour? Chacun selon ses inclinations donnera sa réponse. Notons quand même que cette idée, une fois débarrassée de ses présupposés idéologiques, annonce lointainement le si performant programme européen ERASMUS dont l'énorme succès confirme bien que la jeunesse, pour se former, apprécie les voyages et les brassages de cultures.

\section{Bilan du parcours : des voyages enrichissants}

Autant de voyageurs, autant de raisons de voyager, autant de points de vue sur la réussite du voyage. Alexandre, César, Gengis Khan, Hannibal, ont "voyagé ». Les conquêtes sont leurs réussites. Strabon, Hérodote, Marco Polo, Xénophon par procuration, ont parcouru leurs mondes. Leurs récits de voyage sont leurs réussites. En référence à leurs motivations, les héros de cet ouvrage n'ont pas tous, et de loin, « réussi » leurs voyages. Mais celles et ceux, contributeurs du colloque, qui nous en ont relaté les épisodes, les méandres et les aléas proposent ici des rencontres humaines originales et éclairantes, souvent attachantes. L'émotion de l'auteur(e) pointe même parfois sous son apport scientifique. La rigueur académique n'interdit pas l'empathie envers ceux sur la vie desquels on travaille.

Aujourd'hui, pour beaucoup, la réussite d'un voyage tient à l'intensité des relations, même éphémères, que le voyageur a pu vivre lors de son déplacement. Le lecteur de cet ouvrage, s'il ne peut croiser le chemin de la république en marche, toujours orienté vers sa construction, s'est enrichi en croisant le chemin des hommes et des femmes du temps de la république naissante, chacun, chacune, fort et forte d'un idéal qui les transportait. 


\section{NOTES}

1. Charles MONNET, François-Anne DAVID, Triomphe de la République Française, 1801-1802, dessin gravé, reproduit dans Philippe BORDEs (dir.), Musée de la Révolution française, Premières collections, Saint Martin d'Hères,, D. Munier imp, 1985, p. 99.

2. FRAGONARD fils, Copia, République française, gravure à l'eau forte et au pointillé, (salon 1798), reproduite dans Claudette HOULD (dir.), La révolution par la gravure. Les Tableaux historiques de la révolution française, une entreprise éditoriale d'information et sa diffusion en Europe (1791-1817), Lyon, RMN, 2002, p. 260. Catalogue de l'exposition 2002 du musée de la Révolution française de Vizille du 21 juin au 4 novembre 2002.

3. Aimé-Jules DALOU, Le Triomphe de la République, Groupe sculpté en bronze, Paris, Place de la Nation, 1889.

4. Félix CHARPENTIER et Gustave DEBRIE, Monument élevé à la mémoire des Girondins, Groupe sculpté en bronze, Bordeaux, Place des Quinquonces, 1893, Base Mérimée, Architecture, notice PA33000074 et Roger GALY, Roland CASTELNAU, Le monument aux girondins, Bordeaux, Ed. Sous le vent / Stendhal diffusion, 1989, Col. L'esprit des lieux.

5. Les bases philosophiques de cette société parfaite que serait la république sont rappelées par B. BLANCHET, La Cité idéale, dans V. DUCLERT, C. PROCHASSON (dir.), Dictionnaire critique de la république, Paris, Flammarion, 2007, p. 107-115.

6. Gilles BERTRAND, Pierre SERNA, "Pour lire cet ouvrage... ", dans Gilles BERTRAND, Pierre SERNA (dir.), La République en voyage 1770-1830, PUR, Rennes, 2013, p. 37. L'ouvrage, objet de ce compterendu, sera ensuite désigné dans les notes par le terme République.

7. Ibidem.

8. Parfois au prix de quelques contorsions sémantiques, comme par exemple dans l'introduction à la troisième partie, dans laquelle il est affirmé que « la littérature républicaine universalise le possibilisme d'un cosmopolitisme des souverainetés différentes et communes des peuples.» République, p. 183.

9. Ibid., p. 38.

10. Ibid., p. 16.

11. Ibid., p. 30.

12. Daniel ROCHE, Les circulations dans l'Europe moderne, XVII ${ }^{e}-X V I I I^{e}$ siècle, Paris, Fayard/Pluriel, 2010, p. 97. Daniel Roche dont les productions et les abondantes bibliographies, sur le thème initialement nommé Humeurs vagabondes, sont étrangement absentes de La République en voyage.

13. Daniel Roche, Ibid., p. 762-789 et République, par exemple p. 225, 228.

14. C'est pourquoi j'emploie « panel » pour désigner leur groupe, de préférence à «échantillon », trop connoté sondage et scientificité statistique.

15. Jean-René AYMES, «Ferveur républicaine ou modération du diplomate? L'Espagne et les Espagnols dans les éditions successives du Tableau de l'Espagne Moderne de Jean-François Bourgoin ou la remise en cause de plusieurs stéréotypes entre 1788 et 1807 ", dans République, p. 251-264.

16. Daniela TINKOVÁ, «La peste de la liberté. Les Français suspects de jacobinisme en Bohême et en Moravie (1790-1800)», dans République, p. 109-121.

17. Jean Dhombres, «Les « atours » de la science républicaine en voyage à la toute fin du XVIIIe siècle et les effets du retour », dans République, p. 311-330.

18. Guillaume Mazeau, «L'errance républicaine, les Girondins et l'exil intérieur », dans République ,p. 149-162.

19. Nicole Pellegrin, «Errances, odyssées ou cheminements? Quelques religieuses voyageuses au temps des Républiques », dans République, p. 123-147. 
20. Par dépouillement de «l'Index des noms de personnes », dans République, p. 417-432.

21. Comme le montre avec brio Annie Jourdan dans la première contribution du recueil, «La République d'avant la République (1760-1791), Voyages français en terres de liberté », dans République, p. 47-62.

22. Démarche critique souhaitée par exemple par Pierre Serna, «Le pari politique de Brissot ou lorsque le Patriote Français, l'Abolitionniste Anglais et le Citoyen Américain sont unis en une seule figure de la liberté républicaine », La Révolution française, [En ligne], 5 | 2013, URL : http:// lrf.revues.org/1021.

23. Lafayette très malmené dans son propre pays à partir de 1792, et ensuite de façon récurrente, comme le rappelle Elisabeth LIRIS, "Lafayette ou le long périple du « Héros des deux mondes » entre républicanisme et démocratie royale des vrais constitutionnels", dans République, p. 361-375.

24. Jean-Luc CHAPPEY, «La traduction comme pratique politique chez Antoine-Gilbert Griffet de Labaume (1756-1805)», dans République, p. 233-243.

25. Voir échanges et conclusions du séminaire "Politique et traduction" mené à partir du premier semestre 2014 sous la direction de Mme Christine Lombez, Professeur de littérature comparée à l'Université de Nantes, Laboratoire l'AMo, EA 4276.

26. Souligné par l'auteur

27. Lucia BERGAMASCO, «James Madison et les républiques anciennes et modernes : un voyage dans et par les livres », dans République p. 187-196.

28. Gabor GELLÉRI, « Voyager : un programme républicain », dans République, p. 221-231.

29. Daniel ROCHE, « De l'utilité des voyages », dans op. cit., Chapitre II, p. 49-93.

30. Gabor GELLERI, art. cit., p. 231.

\section{AUTEURS}

\section{DOMINIQUE WAQUET}

Docteur en sciences économiques,

Chercheur associé à l'IHRF 